\title{
El género Chrysactinia (Asteraceae, tribu Tageteae) en México
}

\section{The genus Chrysactinia (Asteraceae, tribe Tageteae) in Mexico}

\author{
José Luis Villaseñor* y María del Rosario Redonda-Martínez \\ Departamento de Botánica, Instituto de Biología, Universidad Nacional Autónoma de México. Apartado postal 70-367, 04510 México, D.F., México. \\ *Correspondencia: vrios@ibiologia.unam.mx
}

\begin{abstract}
Resumen. El género Chrysactinia (Asteraceae, Tageteae) está constituido por 6 especies, distribuidas principalmente en el centro y noreste de México, una de ellas alcanza el suroeste de los Estados Unidos. Se presenta una sinopsis del género, una clave para la identificación de las especies y mapas de distribución.
\end{abstract}

Palabras clave: Asteraceae, Chrysactinia, Tageteae, México.

\begin{abstract}
The genus Chrysactinia (Asteraceae, Tageteae) includes 6 species distributed in central and northeastern Mexico, with 1 species extending its distribution to the southwestern United States. A synopsis of the genus is provided, including a key to species identification as well as distribution maps.
\end{abstract}

Key words: Asteraceae, Chrysactinia, Tageteae, México.

\section{Introducción}

El género Chrysactinia A. Gray es uno de los 23 géneros que constituyen la tribu Tageteae, que diversos autores ubican como parte de la tribu Helenieae (Rzedowski, 1978) o Karis y Ryding (1994), quienes la consideran subtribu (Pectidinae) o parte de la tribu Heliantheae (Cronquist 1955; Strother 1999). Sin embargo, estudios recientes (Funk et al., 2005) parecen indicar que sus miembros conforman un grupo monofilético, relacionado con la tribu Helenieae.

Chrysactinia es un grupo característico dentro de la tribu Tageteae; aunque no tiene sinapomorfias que lo definan como monofilético, contiene una serie de características particulares que permiten diferenciar sus miembros de cualquier otro género de la tribu. Comparte con algunos géneros la presencia de un vilano formado por cerdas capilares (Cuadro 1); por otra parte, la forma de crecimiento sufruticosa o arbustiva la comparte con Gymnolaena y algunas especies de Pectis y Porophyllum. En el Cuadro 1 se presenta una clave para diferenciar los géneros de la tribu con vilano formado exclusivamente de cerdas capilares.

Strother (1977) estudia taxonómicamente el género y reconoce su estrecha relación con 2 géneros cubanos, Harnackia Urb. y Lescaillea Griseb. No obstante, éstos se diferencian de los miembros de Chrysactinia por su

Recibido: 01 febrero 2008; aceptado: 29 junio 2008 forma de crecimiento, pues son sufrútices escandentes, con ramas muy largas y algo suberosas, condiciones nunca observadas en Chrysactinia. Strother (1977) los relaciona con Chrysactinia por la semejanza en la forma de las cabezuelas y del vilano; además, por la morfología de las hojas, muy semejante a la observada en $C$. pinnata. Sin duda es necesario un estudio integral de este complejo para esclarecer la monofilia de Chrysactinia y la taxonomía del mismo, pero debido a la carencia de material herborizado en herbarios mexicanos de los géneros cubanos, se ha preferido dejar para otro estudio la discusión de la circunscripción taxonómica del complejo. En este trabajo sólo se presenta una sinopsis de las especies del género, que en su totalidad se encuentran en México.

En 1977, cuando Strother publicó la revisión del género Chrysactinia, se conocían 5 especies. Sin embargo, Jerzy Rzedowski y Graciela Calderón (1998) publicaron una adicional, por lo que en la actualidad el género está constituido por 6 especies, pertenecientes a 3 secciones, si se acepta la clasificación propuesta por Strother (Cuadro 2). Son endémicas de México 5 especies (Figs. 1, 2 y 3) y solamente 1 (C. mexicana) se extiende hacia Nuevo México y Texas, estados vecinos de los Estados Unidos.

Chrysactinia mexicana presenta la más amplia distribución, pues se ha registrado en 16 estados (Fig. 4), C. pinnata y C. truncata se registran en 4 estados (Figs. 2 y 3); C. lehtoae, de Sinaloa y C. luzmariae de Guanajuato presentan distribución restringida a un solo estado, ambas se conocen solamente de la localidad tipo. 
Cuadro 1. Clave para diferenciar a los géneros de la tribu Tageteae relacionados con el género Chrysactinia por la presencia de un vilano de cerdas capilares

1. Cabezuelas homógamas, flores radiadas (liguladas) ausentes.

2. Hierbas o en ocasiones sufrútices erectos; hojas generalmente alternas; tallo no suberoso.

2. Sufrútices trepadores; hojas opuestas; tallo suberoso; plantas conocidas de Cuba.

1. Cabezuelas heterógamas, flores radiadas (liguladas) presentes.

3. Hierbas anuales o perennes.

4. Base de las hojas con 2 o más pares de cerdas marginales.

4. Base de las hojas sin cerdas marginales.

5. Hojas divididas; flores radiadas blancas, con tintes rojizos o rosa.

5. Hojas simples; flores radiadas blancas o amarillas, sin tintes de otro color.

6. Hojas lineares; flores radiadas blancas; plantas de ambientes xerofíticos.

6. Hojas lanceolado-elípticas; flores radiadas amarillas; plantas acuáticas.

3. Arbustos o sufrútices.

7. Base de las hojas con 2 o más pares de cerdas marginales.

7. Base de las hojas sin cerdas marginales.

8. Plantas trepadoras; tallo suberoso; plantas conocidas de Cuba.

8. Plantas erectas; tallo no suberoso.

Porophyllum Adans. Lescaillea Griseb.

Pectis L.

Nicolletia A. Gray

Leucactinia Rydb. Hydropectis Rydb.

Pectis L.

Harnackia Urb. Chrysactinia A. Gray
Cuadro 2. Secciones (Strother 1977) donde se ubican las especies de Chrysactinia

\author{
Chrysactinia A. Gray sección Chrysactinia \\ 1. Chrysactinia acerosa S.F. Blake \\ 2. Chrysactinia mexicana A. Gray
}

Chrysactinia A. Gray sección Phylloloba S.F. Blake

3. Chrysactinia pinnata S. Watson

4. Chrysactinia truncata S. Watson

5. Chrysactinia luzmariae Rzed. et Calderón

Chrysactinia A. Gray sección Tagetifolia Strother 6. Chrysactinia lehtoae D.J. Keil

\section{Materiales y método}

Se estudió material herborizado que se encuentra depositado en diversos herbarios nacionales y del extranjero: Herbario Nacional de México (MEXU), Herbario de la Universidad Autónoma de San Luis Potosí (SLPM), Herbario del Instituto de Ecología en Xalapa (XAL) y, de manera parcial, la colección del Herbario de la Universidad de Texas (TEX). Se evaluaron diversos caracteres morfológicos que se consideraron de importancia taxonómica y útiles para la correcta identificación de las especies, elaborándose una clave de identificación. Las localidades de recolecta fueron georreferenciadas y se elaboraron mapas de distribución para cada especie y para el género. En los mapas de distribución (Figs. 1-5), los sitios de recolecta (puntos) están unidos mediante una red de tendido mínimo para ilustrar el trazo biogeográfico del género y las especies.

\section{Descripción}

Chrysactinia A. Gray, Mem. Amer. Acad. Arts, ser. 2. 4: 90. 1849.

Arbustos o sufrútices, $10-80 \mathrm{~cm}$ alto. Tallos erectos, glabros o pubescentes. Hojas opuestas o alternas, simples, acerosas a lineares o pinnadamente divididas, con 720 lóbulos lineares a ovados, con pocas a numerosas glándulas oleíferas pelúcidas, distribuidas en la lámina de la hoja. Cabezuelas heterógamas, radiadas, pedunculadas, solitarias; pedúnculos glabros a pubescentes, usualmente bracteolados. Involucro turbinado, campanulado o hemisférico, 3-8 mm largo. Brácteas involucrales 8-13, uniseriadas, lineares a lanceoladas, con los márgenes escariosos, usualmente carinadas, persistentes, con una glándula oleífera pelúcida subapical y 2-4 glándulas basales. Receptáculo alveolado, desnudo o rara vez conteniendo unas cuantas páleas delgadas y deciduas (C. mexicana). Flores radiadas 8-13, pistiladas, fértiles, corolas amarillas o anaranjadas; flores del disco 12-70, hermafroditas, fértiles, corolas amarillas o en ocasiones verdosas, cilíndricoinfundibuliformes. Aquenios cilíndricos o fusiformes, negros o pardos, estriados, hispídulos, 2-4 mm largo. Vilano formado por 20-40 cerdas libres, dispuestas en una sola serie, iguales o subiguales, 3.5-6.0 mm largo, más largas que el aquenio.

Género constituido por 6 especies, casi todas endémicas de México. El grupo se distribuye principalmente hacia la porción este del país (Fig. 5), con una sola especie (C. lehtoae) registrada en su porción noroeste (Fig. 2). Tomando como referencia a Ferrusquía-Villafranca (1990) que subdivide México en provincias bióticas morfotectónicas, Chrysactinia se registra en 8, destacando 


\section{Clave para identificar las especies de Chrysactinia en México}

1. Hojas simples, lámina linear, $2 \mathrm{~mm}$ de ancho o menos.

2. Hojas opuestas, acerosas (Fig. 6a); lámina $1 \mathrm{~mm}$ o menos de ancho; brácteas involucrales por lo general 8.

C. acerosa

2. Hojas alternas, lineares (Fig. 6b); lámina 1-2 mm ancho; brácteas involucrales por lo general 13.

C. mexicana

1. Hojas pinnado-lobuladas, lóbulos de la lámina rara vez lineares, por lo general $3 \mathrm{~mm}$ o más de ancho.

3. Lóbulos de la hoja linear-lanceolados (Fig. 6c); plantas conocidas de Sinaloa.

C. lehtoae

3. Lóbulos de la hoja cuneados a ovados.

4. Lóbulos de la hoja con el ápice truncado (Fig. 6f).

C. truncata

4. Lóbulos de la hoja con el ápice agudo a mucronato.

5. Hojas con los lóbulos alcanzando casi la vena media (Fig. 6e); ápice de la hoja agudo; pedúnculos pilosos.

5. Hojas con los lóbulos sin alcanzar la mitad de la lámina hacia la vena media (Fig. 6d); lóbulos de la hoja con el ápice mucronato; pedúnculos glabros.

C. pinnata

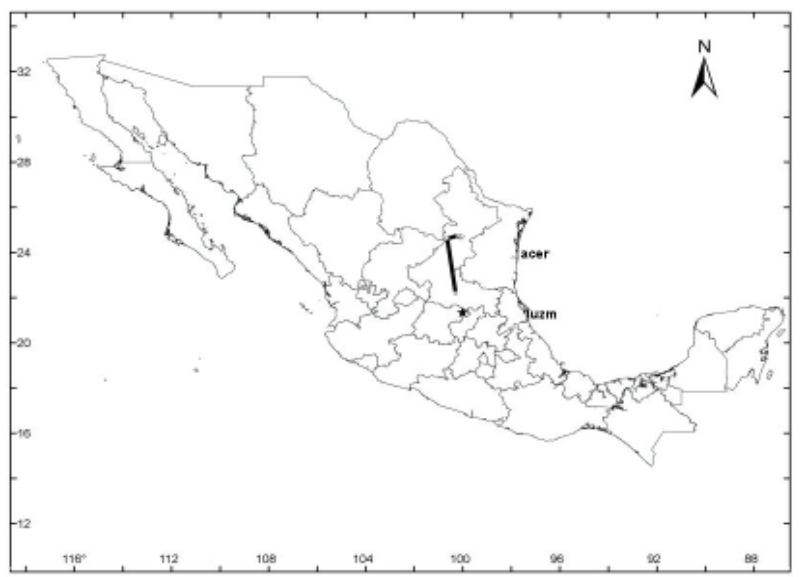

Figura 1. Distribución de Chrysactinia acerosa (acer) y $C$. luzmariae (luzm).

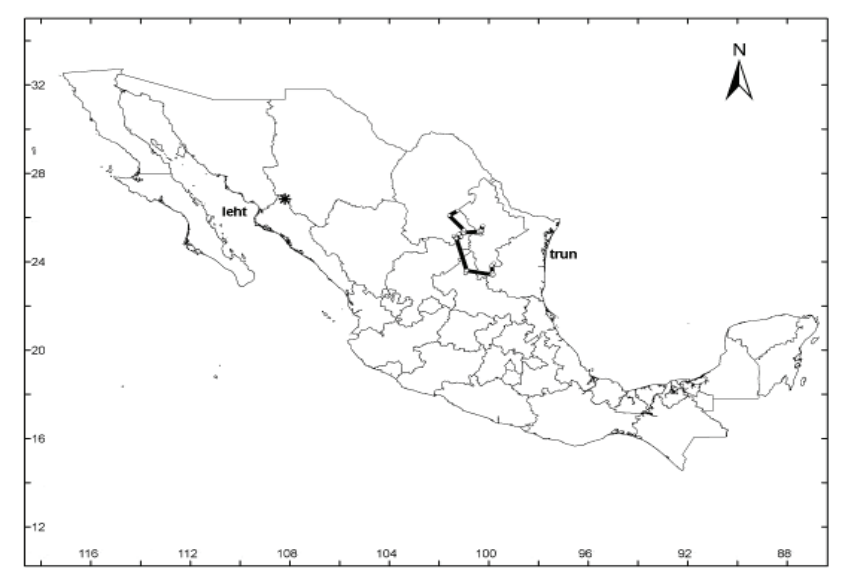

Figura 2. Distribución de Chrysactinia lehtoae (leht) y $C$. truncata (trun).

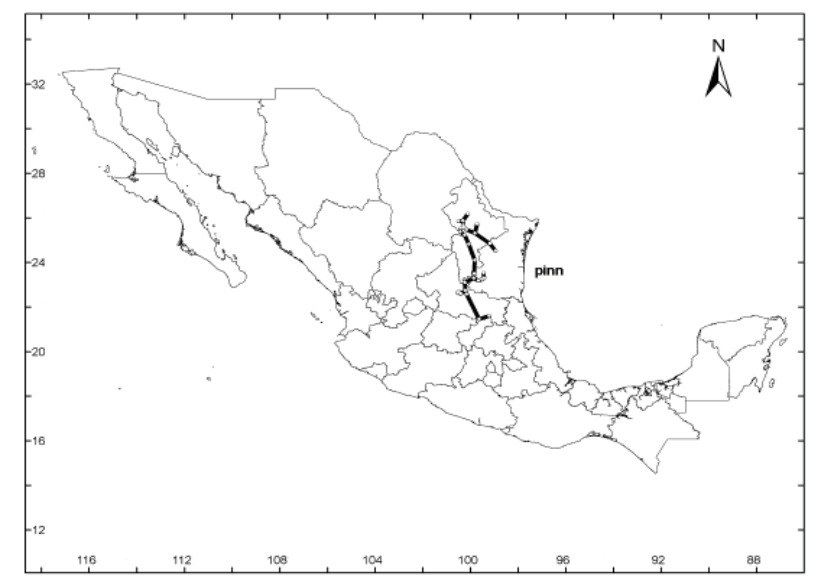

Figura 3. Distribución de Chrysactinia pinnata (pinn).

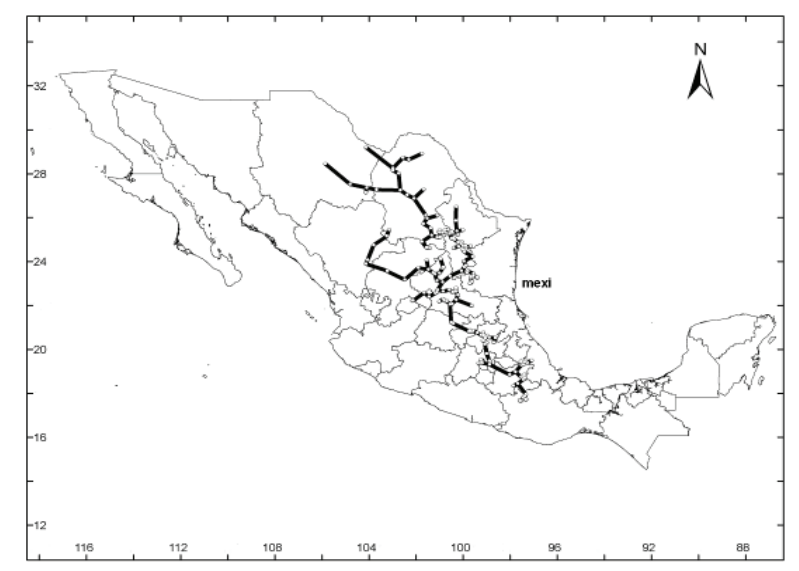

Figura 4. Distribución de Chrysactinia mexicana (mexi) en México. 


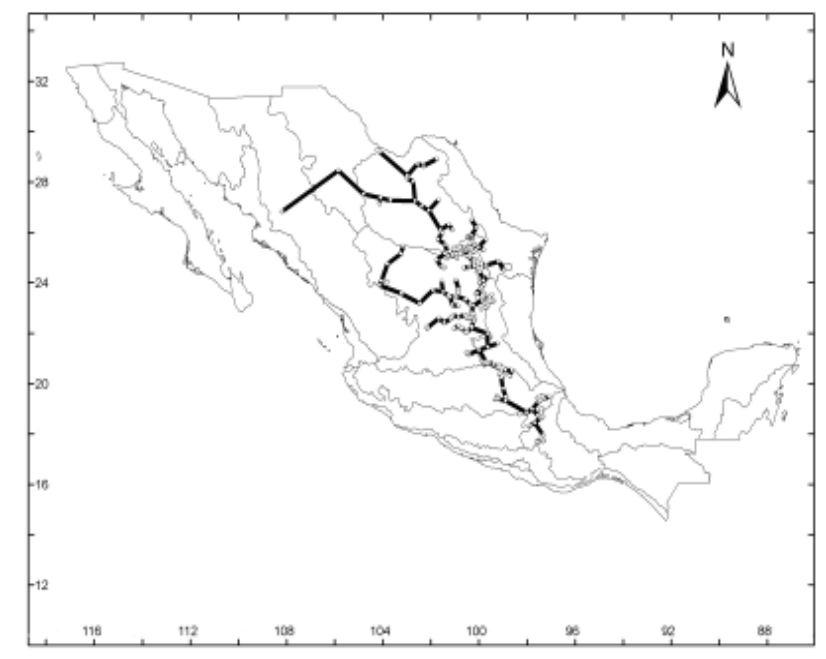

Figura 5. Distribución del género Chrysactinia en México mostrada sobre el mapa de las provincias morfotectónicas de México.

la provincia sierra Madre orientalense, donde se registran 5 especies, seguida de la provincia altiplanense con 4 y la coahuilense con 3. Una sola especie se registra en las provincias chihuahuense, neovolcanense, oaxacense, sierra Madre occidentalense y tamaulipense.

El género predomina en comunidades vegetales propias de ambientes xerófilos y templados. Los chaparrales del noreste de México, así como los bosques de pino-encino son las comunidades que registran el mayor número de especies (4); con menor número están los bosques de encinos y de pinos, y los matorrales xerófilos (3). El intervalo altitudinal donde se le encuentra es muy amplio, desde los $350 \mathrm{~m}$ de altitud hasta los $2950 \mathrm{~m}$.

\section{Resumen taxonómico}

Chrysactinia acerosa S.F. Blake, Proc. Amer. Acad. Arts 51: 524. 1916 (Fig. 7).

Arbustos, 10-20 cm alto. Tallos glabros o pubescentes. Hojas opuestas, simples, acerosas, 4-12 mm largo, 0.2-0.5 $\mathrm{mm}$ ancho, algunas veces algo suculentas. Pedúnculos pubescentes, con 1-5 bractéolas. Involucro turbinado a campanulado, 3-4 mm largo. Brácteas involucrales por lo general 8, lineares, con 1 glándula pelúcida subapical. Receptáculo desnudo. Flores radiadas 7-9, amarillas; flores del disco 12-15, amarillas. Aquenios alrededor de $2 \mathrm{~mm}$ largo, cilíndricos, negros. Cerdas del vilano 20-30, 4-5 mm largo.

Hábitat. En vegetación halófila y matorrales xerófilos, en altitudes entre 1780 y $2100 \mathrm{~m}$.
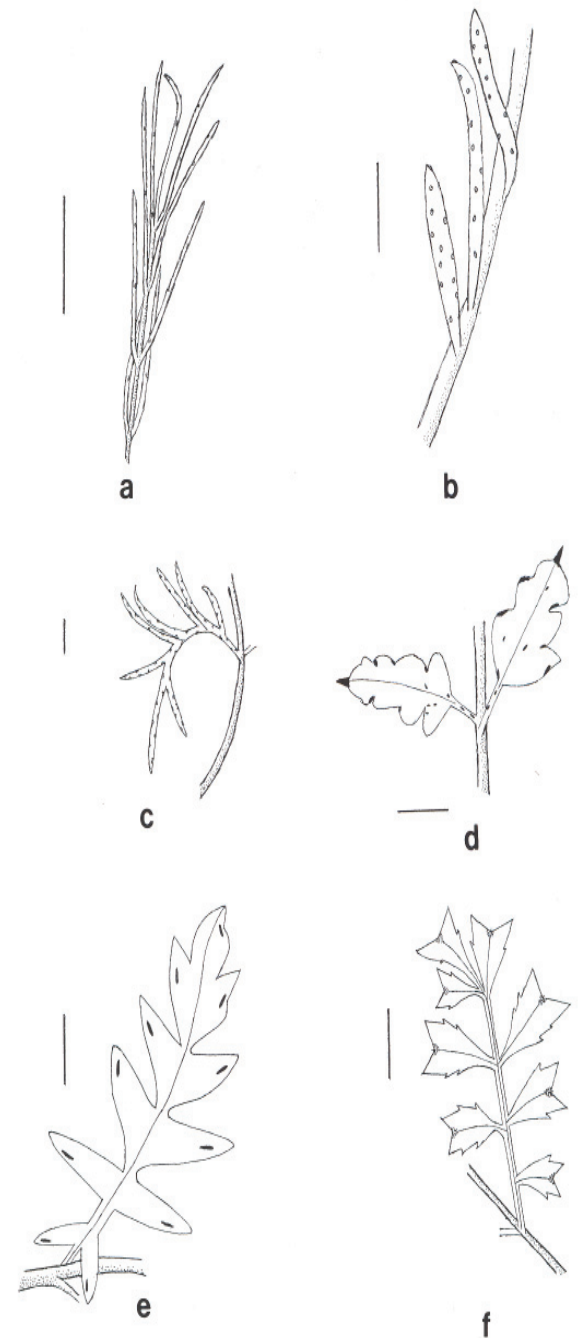

f

Figura 6. Variación morfológica de las hojas en el género Chrysactinia. a, C. acerosa; b, C. mexicana; c, C. lehtoae; d, C. luzmariae; e, C. pinnata; f, C. truncata. Escala: $5 \mathrm{~mm}$.

Floración. De junio a octubre.

Distribución. Endémica de México (Fig. 1): NUEvo LEón: Hinton 22178 (HINTON, MEXU, TEX); Johnston 8019 (MEXU, TEX); Sundberg 1827 (MEXU, TEX); Turner 6321 (ENCB, MEXU, TEX); san LUIS Potosí: Purpus 5136 (GH fotografía en ENCB, MEXU, NY fotografía en MEXU). 


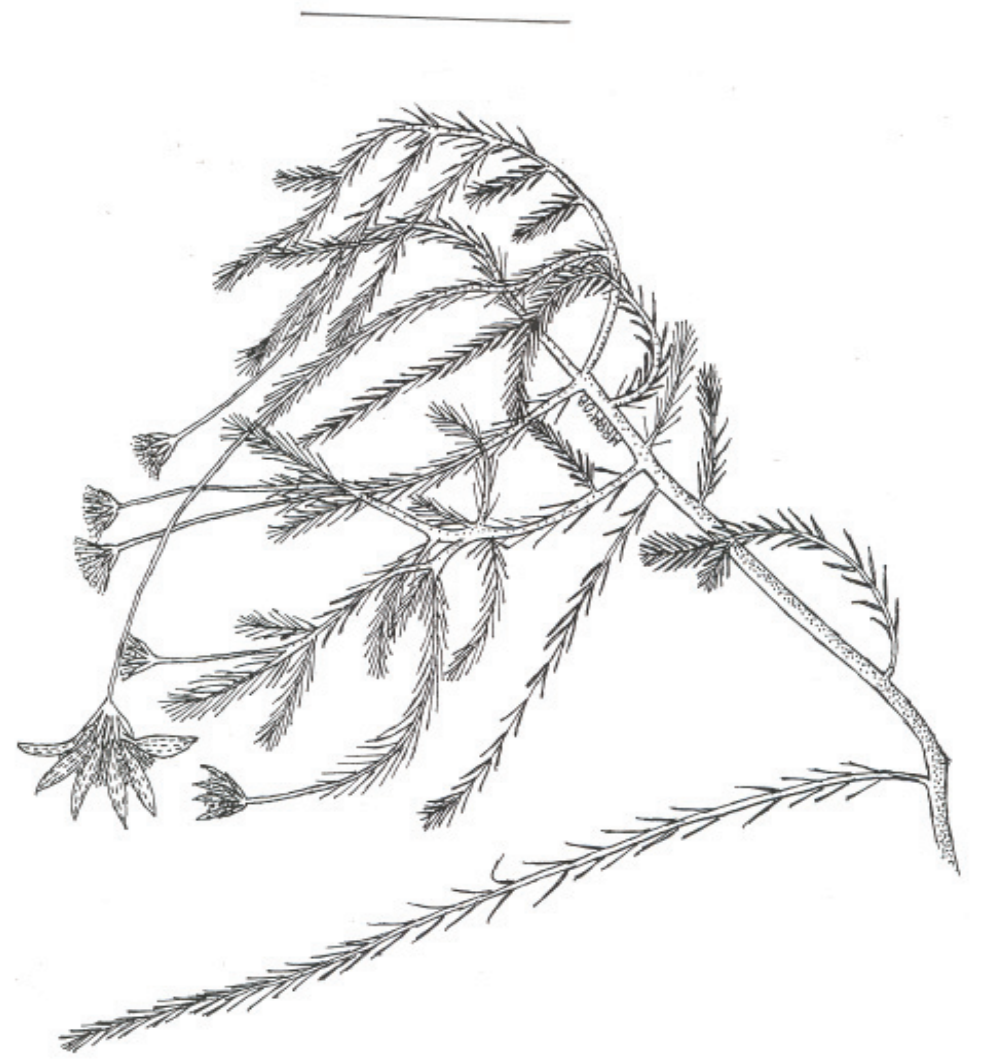

Figura 7. Chrysactinia acerosa. Rama con cabezuelas (basado en Sundberg 1827 MEXU). Escala: $3 \mathrm{~cm}$

Chrysactinia lehtoae D. J. Keil, Madroño 23: 374. 1976. Arbustos, ca. $30 \mathrm{~cm}$ alto. Tallos pubescentes, los tricomas ferrugíneos. Hojas opuestas, pinnado-lobuladas, 7-9 lóbulos linear-lanceolados, 12-20 mm largo, 0.5-1.8 mm ancho. Pedúnculos glabros, con 2-3 bractéolas. Involucro turbinado, alrededor $5 \mathrm{~mm}$ largo. Brácteas involucrales 13, lineares, con 1 glándula pelúcida subapical y 2-4 glándulas pelúcidas basales. Receptáculo desnudo. Flores radiadas 12-13, amarillas; flores del disco alrededor de 40, amarilloverdosas. Aquenios alrededor de $3 \mathrm{~mm}$ largo, cilíndricos, negros. Cerdas del vilano 25-30, 4-5 mm largo.

Hábitat. En bosque de pino-encino, alrededor de los 1300 m de altitud.

Floración En noviembre.

Distribución. Endémica de México (Fig. 2) SINALOA: Lehto 19551 (ASU).

Ilustración. Madroño 23: 375. 1976.

Chrysactinia luzmariae Rzed. et Calderón, Bol. Inst. Bot. Univ. Guadalajara 5: 61. 1998.

Sufrútices, $20-50 \mathrm{~cm}$ alto. Tallos glabros o pubescentes. Hojas opuestas, pinnado-lobuladas, 8-12 lóbulos lanceolados, ápice mucronato, 10-15 mm largo, 3-10 mm ancho. Pedúnculos glabros, con 1-4 bractéolas. Involucro campanulado, 6-8 mm largo. Brácteas involucrales 8, lanceoladas, con 1 glándula pelúcida subapical y 2 glándulas pelúcidas basales. Receptáculo desnudo. Flores radiadas 8, amarillas; flores del disco 25-30, amarillas. Aquenios 2.5-3.0 mm largo, cilíndricos, pardos. Cerdas del vilano 25-30, 4-5 mm largo.

Hábitat. En chaparrales, a una altitud de alrededor de 900 m.

Floración. En julio.

Distribución. Endémica de México (Fig. 1): GuanaJuato: Rzedowski 53447 (IEB, MEXU).

Ilustración. Bol. Inst. Bot. Univ. Guadalajara 5: 63. 1998.

Chrysactinia mexicana A. Gray, Mem. Amer. Acad. Arts, n. s., 4: 93. 1849.

Pectis taxifolia Greene, Leafl. Bot. Observ. Crit. 1: 148. 1905.

Arbustos, $20-40 \mathrm{~cm}$ alto. Tallos glabros a pubescentes. Hojas alternas, simples, lineares, 5-10(-23) mm largo, 1$2 \mathrm{~mm}$ ancho. Pedúnculos glabros a pubescentes, con 1-5 
bractéolas. Involucro turbinado a hemisférico, 3.5-5.0 mm largo, 1-2 mm ancho. Brácteas involucrales por lo general 13(-14), lineares o lanceoladas, con 1 glándula pelúcida subapical y 2-4 glándulas pelúcidas basales. Receptáculo con unas cuantas páleas delgadas y deciduas. Flores radiadas (8-)13, amarillas, en ocasiones amarillo-verdosas; flores del disco 25-40, amarillas. Aquenios 3-4 mm largo, cilíndricos, negros. Cerdas del vilano 30-40, 3.5-5.0 mm largo.

Hábitat. En bosque de encino, bosque de Juniperus, bosque de pino, bosque de pino-encino, bosque tropical caducifolio, chaparral, matorrales xerófilos, pastizal, vegetación halófila, vegetación riparia y como ruderal en ambientes perturbados, en altitudes que van desde los 750 a los $2875 \mathrm{~m}$.

Floración. En todos los meses del año.

Distribución. Estados Unidos (Nuevo México y Texas) y México (Fig. 4): Aguascalientes: de la Cerda 1046 (HUAA); García 2853 (HUAA); García 3543 (HUAA); McVaugh 23644 (ENCB, LL-TEX); Medellín s. n. (SLPM); Rzedowski 25001 (ENCB); Siqueiros 3616 (HUAA); CHIHUAHUA: Johnston 8969 (MEXU, TEX); Johnston 9020 (MEXU); Gentry 23208 (MEXU); Pringle 6 (MEXU); Stewart 903 (LL-TEX, MEXU); coAHUILA: Arredondo 42 (XAL); Avila s. n. (ENCB); Bridges 13067 (MEXU, TEX); Carranza 941 (MEXU); Carranza 1153 (MEXU); Castillo 532 (MEXU); Johnston 7551 (LL-TEX, MEXU); Johnston 9088 (LL-TEX, MEXU); Johnston 9419 (MEXU, TEX); Dunn 22491 (ENCB); Ely 147 (XAL); Ely 202 (XAL); Encina 1111 (IBUG); Freeman 2044 (MEXU); Fuentes s. n. (ENCB); García 1014 (ENCB); Gregg 103 (K fotografía en MEXU); Gregg 656 (GH fotografía en ENCB); Hartman 3993 (ENCB); Henrickson 13144 (LL-TEX, MEXU); Hinton 16567 (ENCB, MEXU); Hinton 16629 (ENCB, MEXU); Lyonnet 3529 (MEXU); Lyonnet 3814 (MEXU); ND 9318 (MEXU); Marroquín s. n. (ENCB); Palmer 63 (MEXU); Palmer 275a (MEXU); Palmer 287 (MEXU); Passini 4062 (ENCB); Passini 4086 (ENCB); Passini 4234 (ENCB); Passini 4448 (ENCB); Passini 5161 (ENCB); Pinkava 6001 (ENCB); Pringle 2641 (MEXU); Robert 133 (ENCB); Robert 205 (ENCB); Robert 5655 (ENCB); Rodríguez 829bis (ENCB); Rodríguez 1336 (MEXU); Seigler 9278 (MEXU); Snow 6725 (MEXU, TEX); Stanford 25 (LL-TEX, MEXU); Stanford 218 (MEXU); Valdés 1329 (SLPM), Valdés 1577 (SLPM, TEX, XAL); Villarreal 3687 (SLPM); Villarreal 5377 (XAL); Villaseñor 1275 (MEXU); Villaseñor 1288 (MEXU); Winckley s. n. (MEXU); DISTRITO FEDERAL: Lyonnet 1553 (ENCB, MEXU); Lyonnet 2283 (ENCB, MEXU); Pringle 9324 (MEXU); DURANGo: Chávez 92 (CHAPA, CIIDIR, IBUG, MEXU, OAX); Diggs 3064 (TEX, XAL); González 1669 (CIIDIR, ENCB, HUAA,
MEXU, TEX); GUANaJUATO: Rzedowski 41685 (ENCB, IBUG, MEXU); Ventura 7209 (MEXU, XAL); Ventura 9658 (MEXU); hidAlgo: Finney s. n. (MEXU); González 2525 (ENCB); González 3522 (ENCB); González 3768 (MEXU); González 8874 (MEXU); González 9636 (MEXU); Hiriart 138 (MEXU, XAL); Hernández 3676 (MEXU); Hernández 6178 (ENCB, MEXU); Hernández 6440 (MEXU, XAL); Hess 4657 (MEXU); López 134 (XAL); Martínez 19 (MEXU); Miranda 9894 (MEXU); Paray 2372 (ENCB); Rangel 37 (ENCB); Urbina $s$. n. (MEXU); Velasco 8 (MEXU); MÉXICO: García s. $n$. (ENCB, MEXU, SLPM); Medellín 429 (ENCB, MEXU); Rzedowski 24082 (ENCB, LL-TEX); Rzedowski 34416 (ENCB, MEXU, XAL); Salazar s. n. (MEXU); Urbina s. n. (MEXU); NUEvo LEón: Cochrane 8444 (ENCB, MEXU); Cowan 3822 (MEXU, SLPM, TEX); Cowan 4630 (MEXU, TEX); Cronquist 11291 (LL-TEX, MEXU); Díaz s. n. (ENCB); Dorr 2323 (MEXU, TEX); Dorr 2641 (MEXU, TEX); Dorr 2649 (ENCB, MEXU, TEX); Estrada s. n. (MEXU); Estrada 928 (MEXU); Estrada 956 (MEXU); González 4967 (MEXU); Gregg s. n. (NY); Hinton 17756 (ENCB, HINTON, IBUG, MEXU, TEX, XAL); Hinton 18677 (ENCB, HINTON, IBUG, MEXU, SLPM, TEX, XAL); Hinton 19610 (CHAPA, HINTON, MEXU, TEX); Johnston 4235 (MEXU); Mueller 237 (MEXU, TEX); ND s. n. (MEXU); Nesom 4305 (ENCB, MEXU, TEX); Passini 6527 (ENCB); Puig 6907 (ENCB); Ramírez s. n. (XAL); Sánchez 3738 (MEXU); Seigler 3205 (MEXU); Sundberg 3120 (MEXU, TEX); Valdés 564 (ENCB); Yahara 1559 (MEXU); Yahara 1767 (MEXU); OAXACA: Campos 5222 (MEXU); Chiang 2529 (MEXU, TEX); Cruz 1987 (ENCB); Cruz 2062 (ENCB); García 2507 (MEXU); Rzedowski 34878 (ENCB); Tenorio 20248 (MEXU, TEX); Turner 80 (MEXU); Ventura 15444 (ENCB, MEXU); PUEBLA: Arsene 2207 (MEXU); Arsene 2248 (MEXU); Cabrera 11942 (MEXU); Calzada 4702 (ENCB, XAL); Castillo 18053 (MEXU); Chiang 222 (HUAA, MEXU); García 4073 (MEXU); García 7089 (MEXU); González 964 (MEXU); Mares 2 (XAL); Mayfield 911 (MEXU, TEX); Ramos 103 (MEXU, XAL); Robert 86 (ENCB); Rodríguez 65 (MEXU); Rodríguez 292 (XAL); Salinas 3467 (MEXU); Sandoval 309 (ENCB, XAL); Segura 187 (MEXU, XAL); Takaki s. n. (SLPM); Tenorio 11549 (MEXU, TEX); Tenorio 14132 (MEXU); Tenorio 18286 (MEXU, OAX); Tenorio 21067 (MEXU); Valiente 1113 (MEXU); Vázquez 1965 (ENCB); Ventura 1571 (ENCB, LL-TEX); Ventura 8364 (ENCB, XAL); Ventura 22331 (ENCB, MEXU, XAL); Villaseñor 73 (MEXU, TEX); Webster 20056 (MEXU); QUERÉTARO: Paray 109 (ENCB); Zamudio 2376 (ENCB, IBUG, MEXU); Zamudio 2468 (MEXU, XAL); Zamudio 2750 (MEXU); Zamudio 2778 (MEXU, XAL); Zamudio 3025 
(ENCB); SAN LUIS potosí: Arredondo s. n. (MEXU); Balleza 10270b (HUAZ, MEXU); Ballín s. n. (ENCB, SLPM); Barry 3280 (MEXU); Cárdenas s. n. (SLPM); Chemin 49 (MEXU); Chemin 92 (MEXU); de la Cerda 2610 (HUAA); Diggs 2471 (ENCB, TEX, XAL); García 1316 (ENCB, TEX); Gómez 536 (ENCB, IBUG, SLPM); Gómez 991 (SLPM); Jasso s. n. (SLPM); Lundell 5155 (LL-TEX, MEXU); Marroquín s. $n$. (ENCB); Medellín s. n. (ENCB); Medellín s. n. (SLPM); Medellín 1291 (ENCB, SLPM); Nee 24521 (TEX, XAL); Parada 9 (MEXU); Pérez 3088 (MEXU, XAL); Prigge 3280 (ENCB); Puig 6830 (ENCB, MEXU); Rzedowski 4105 (ENCB, SLPM); Rzedowski 4961 (SLPM); Rzedowski 5523 (SLPM); Rzedowski 6311 (ENCB, MEXU, SLPM); Rzedowski 8823 (ENCB, SLPM); Salas 310 (SLPM); Salas 518 (CHAPA, ENCB, MEXU, SLPM); Salas 614 (MEXU, SLPM); Salas 751 (IBUG, SLPM); Sánchez 2167 (XAL); Schaffner 331 (MEXU); Torres 14548 (MEXU, SLPM); Torres 14638 (MEXU); Torres 15258 (MEXU, SLPM); Torres 15268 (MEXU); TAMaUlipas: Díaz 307 (MEXU, TEX); González 223 (MEXU); González 3498 (MEXU); González 3823 (ENCB); González 4760 (MEXU); González 7036 (MEXU); González 8651 (MEXU); González 8661 (MEXU); González 10020 (MEXU); González 10111 (MEXU); Hess 4716 (MEXU); Jiménez 103 (MEXU, SLPM); Martínez 392 (MEXU); Martínez 456 (ENCB, XAL); Mora 963 (MEXU); Nesom 5960 (MEXU, TEX); Puig 2343 (MEXU); Puig 4755 (ENCB, MEXU); Romo 41 (SLPM); Romo 641 (ENCB, SLPM, XAL); Yáñez 49 (MEXU); Yáñez 186 (MEXU); verAcruz: Acosta 1998 (MEXU); Cronquist 10379 (MEXU, XAL); Martínez 1015 (XAL); Nevling 1074 (MEXU, XAL); Nevling 1743 (MEXU, XAL); Ortega 554 (XAL); Robledo 185 (MEXU, XAL); Rodríguez 45 (MEXU); Turner 15427 (TEX, XAL); Vázquez 2096 (MEXU, XAL); zaCATECAS: Balleza 2841 (HUAZ); Balleza 5304 (HUAZ, MEXU); Balleza 5443 (HUAZ); Balleza 5515b (HUAZ); Balleza 5520 (HUAZ, MEXU); Balleza 6217 (HUAZ); Balleza 10243 (HUAZ); García 307 (ENCB, MEXU); Kalkbiotop s. n. (MEXU); Pennell 17425 (MEXU); Puig 6587 (ENCB); Stanford 494 (MEXU).

Ilustración. Flora Novo-Galiciana 12: 222.1984.

Chrysactinia pinnata S. Watson, Proc. Amer. Acad. Arts 25: 154. 1890 (Fig. 8).

Arbustos o sufrútices, $40-80 \mathrm{~cm}$ alto. Tallos glabros a pubescentes, en ocasiones los tricomas ferrugíneos. Hojas opuestas, pinnado-lobuladas 8-12(-20) lóbulos, lanceolados a elípticos, ápice agudo, 10-25 mm largo, 6-12 mm ancho. Pedúnculos pilosos, con 3-5 bracteolas. Involucro turbinado, 6-8 mm largo. Brácteas involucrales 8 , lineares a lanceoladas, con 1 glándula pelúcida subapical

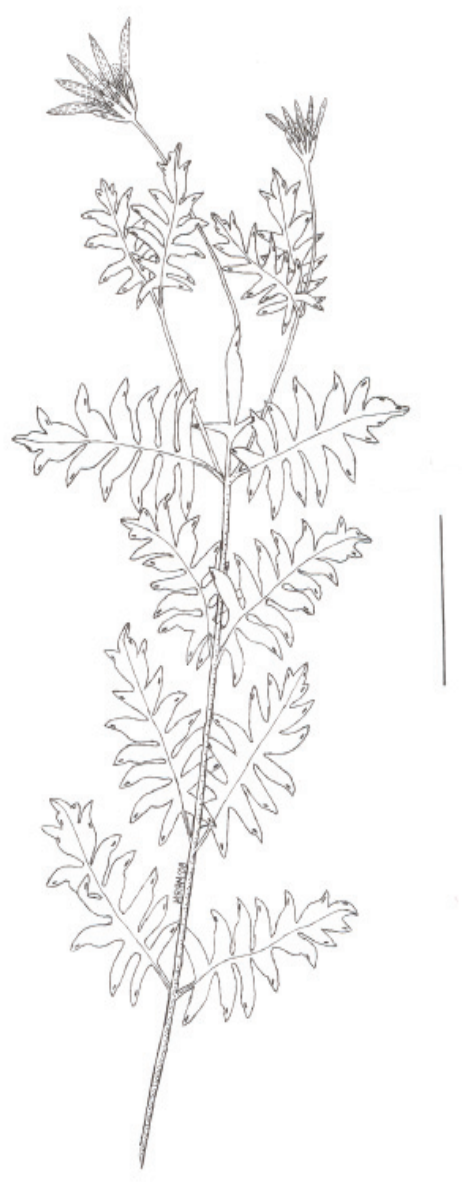

Figura 8. Chrysactinia pinnata. Rama con cabezuelas (basado en González 10198 MEXU). Escala: $3 \mathrm{~cm}$.

y 1-2 glándulas pelúcidas basales. Receptáculo desnudo. Flores radiadas 8, amarillas; flores del disco 20-30, amarillo-anaranjadas. Aquenios 3-4 mm largo, cilíndricos a fusiformes, negros. Cerdas del vilano alrededor de 40, 4-5 mm largo.

Hábitat. En bosque de encino, bosque de pino, bosque de pino-encino, bosque tropical caducifolio, chaparral, matorrales xerófilos y vegetación riparia, desde los 350 a los $1900 \mathrm{~m}$ de altitud.

Floración. En todos los meses del año.

Distribución. Endémica de México (Fig. 3): NUEvo LEón: Carlson 2696 (MEXU, TEX); Hinton 19232 (ENCB, HINTON, MEXU, TEX); Hinton 19973 (HINTON, MEXU); Johnston 5458(MEXU); Marroquín 910 (SLPM); Mueller 275 (MEXU, TEX); Mueller 2043 (MEXU); 


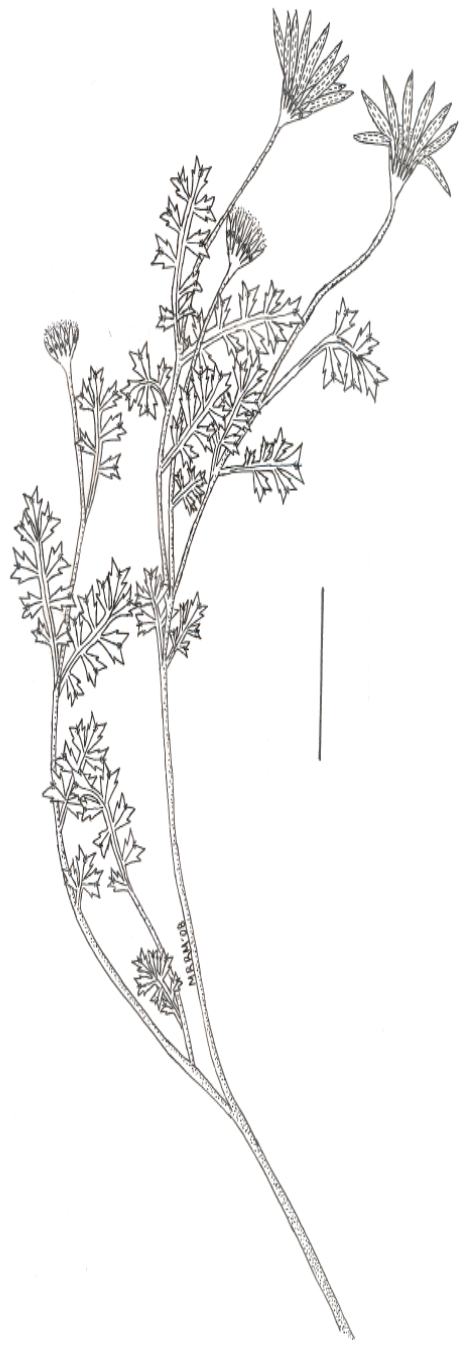

Figura 9. Chrysactinia truncata. Rama con cabezuelas (basado en Lyonnet $3868 \mathrm{MEXU}$ ). Escala: $3 \mathrm{~cm}$.

Pennell 16840 (MEXU); Pringle 2524 (GH fotografía en ENCB, K fotografía en MEXU, MEXU, NY); Sánchez 134 (MEXU); Valdés 304 (ENCB); Valdés 1868 (IBUG); Villarreal s. $n$. (ENCB); Villarreal 9055 (MEXU); Yahara 1733 (MEXU); QUERÉTARo: Carranza 2570 (MEXU, TEX); López 757 (MEXU, XAL); SAN LUIS POTOSí: Gómez 1311 (MEXU, SLPM); Robert 6813 (ENCB); Rzedowski 5586 (SLPM); Rzedowski 6800 (ENCB, MEXU, SLPM); Torres 14516 (MEXU, SLPM); Torres 14924 (MEXU, SLPM); Torres 15435 (MEXU); Torres 15476 (MEXU); TAMaUlipas: González 583 (MEXU); González 4563 (MEXU); González 4750 (MEXU); González 9680 (MEXU); González 10198 (ENCB, MEXU); Hernández
3160 (MEXU); Henrickson 19105 (MEXU, TEX); Martínez 2336 (MEXU); Nesom 6189 (MEXU, TEX); Villaseñor 510 (MEXU).

Chrysactinia truncata S. Watson, Proc. Amer. Acad. Arts 25: 154.1890 (Fig. 9)

Arbustos o sufrútices, ca. $30 \mathrm{~cm}$ alto. Tallos glabros a pubescentes. Hojas opuestas, pinnado-lobuladas, 37(-13) lóbulos cuneados a ovados, ápice truncado, 6-15 mm largo, 4-8 mm ancho. Pedúnculos glabros, con 3-5 bracteólas. Involucro turbinado, 4-6 mm largo. Brácteas involucrales 13, lineares a lanceoladas, con 1 glándula pelúcida subapical y 1-2 glándulas pelúcidas basales. Receptáculo desnudo. Flores radiadas 13, amarillas; flores del disco 35-50, amarillas. Aquenios 2.8-3.9 mm largo, cilíndricos a fusiformes, negros. Cerdas del vilano 25-30, 4-6 mm largo.

Hábitat. En bosque de encino, bosque de pino, bosque de pino-encino, chaparral, matorrales xerófilos y vegetación riparia, entre los 1250 y 2950 m de altitud.

Floración. De mayo a diciembre.

Distribución. Endémica de México (Fig. 2): coahuila: Arce 540 (ENCB, MEXU); Carranza 1615 (MEXU); Johnston 11688 a (LL-TEX, MEXU); Johnston 12063 (LLTEX, MEXU); Lyonnet 3604 (ENCB, MEXU); Lyonnet 3868 (MEXU); Palmer 275a (MEXU); Pringle 3463 (MEXU); Snow 6746 (LL-TEX, MEXU); Stanford 310 (MEXU); Villarreal 6501 (MEXU); NUEVo LEóN: Hinton 17569 (ENCB, HINTON, IBUG, MEXU, TEX, XAL); Hinton 24369 (HINTON, MEXU, TEX); Lyonnet 3961 (MEXU); Mayfield 1305 (MEXU, TEX); Pringle 2601 (GH fotografía en ENCB, K fotografía en MEXU, MEXU, NY); Torres 1108 (MEXU); Valdés s. n. (MEXU); Valdés 129 (ENCB); Villarreal 2730 (ENCB, SLPM, TEX); Yahara 1632 (MEXU); SAN LUIS POTOsí: Johnston 11081 (LL-TEX, MEXU); Rzedowski 8151 (ENCB, MEXU, SLPM); TAMAuliPas: González 4714 (MEXU); González 9205 (SLPM); Johnston 11179 (LL-TEX, MEXU); Mora 5013 (MEXU).

\section{Agradecimientos}

A los curadores de los herbarios citados en el texto, por las facilidades brindadas para la consulta del material. A Rosalinda Medina y Fernando Chiang, por la lectura del manuscrito y sus valiosos comentarios y a Enrique Ortiz por la asistencia técnica en diversas facetas del trabajo. La Comisión Nacional para el Conocimiento y Uso de la Biodiversidad (CONABIO; proyecto U004) y la Unidad de Informática para la Biodiversidad (UNIBIO) del Instituto de Biología, UNAM apoyaron con recursos económicos, humanos y logísticos. 


\section{Literatura citada}

Cronquist, A. 1955. Phylogeny and taxonomy of the Compositae. American Midland Naturalist 53:478-511.

Ferrusquía-Villafranca, I. 1990. Provincias bióticas (con énfasis en criterios morfotectónicos). In Regionalización biogeográfica, IV.8.10. Atlas Nacional de México, vol. II, escala 1: 4000 000. Instituto de Geografía, UNAM. México, D. F.

Funk, V. A., J. B. Randall, S. Keeley, R. Chan, L. Watson, B. Gemeinholzer, E. Schilling, J. L. Panero, B. G. Baldwin, N. García-Jacas, A. Sussana y R. K. Jansen. 2005. Everywhere but Antarctica: using a supertree to understand the diversity and distribution of the Compositae. Biologiske Skrifter 55:343-373.
Karis, O. y O. Ryding. 1994. Tribe Helenieae. In Asteraceae: cladistics and classification, K. Bremer (ed.). Timber, Portland. p. 521-558.

Rzedowski, J. 1978. Claves para la identificación de los géneros de la familia Compositae en México. Universidad Autónoma de San Luis Potosí, San Luis Potosí. 143 p.

Rzedowski, J. y G. Calderón de R. 1998. Dos nuevas especies de fanerógamas calcífilas del centro de México. Boletín del Instituto de Botánica de la Universidad de Guadalajara 5:6168.

Strother, J. L. 1977. Taxonomy of Chrysactinia, Harnackia and Lescaillea (Compositae: Tageteae). Madroño 24:129-139.

Strother, J. L., 1999. Compositae, Heliantheae s.l. In Flora of Chiapas, T. F. Daniel (ed.). California Academy of Sciences. San Francisco. 232 p. 